\title{
O ENVELHECIMENTO DA PESSOA COM DEFICIÊNCIA INTELECTUAL (DI) E A REABILITAÇÃO NEUROPSICOLÓGICA
}

Adriana Daiana Oliveira

\section{RESUMO}

Com o aumento da longevidade para toda a população mundial e devido às inúmeras melhorias nos diversos setores da sociedade, também estão vivendo mais as pessoas com deficiência intelectual (DI), que até 30 anos atrás não ultrapassavam a idade de 15 anos. Porém, o fato de estarem vivendo mais não quer dizer que estejam vivendo melhor, pois além de serem discriminadas pela situação de deficiência, agrega-se o fato de serem idosas. Também se observa, nessa população específica, o fato de apresentarem sinais de envelhecimento precoce devido ao aparecimento de doenças comuns em idades mais avançadas na população em geral. Junta-se a esse panorama, a escassez de políticas públicas efetivas de alcance para essas questões. As famílias e as instituições como a Apae têm buscado dar visibilidade a esses aspectos e demandas, oferecendo apoio e propondo soluções para o atendimento desse público específico, mediante cuidados necessários, inclusive durante o processo de envelhecimento. A reabilitação neuropsicológica tem sido indicada como metodologia complementar para promover a independência e a autonomia dos beneficiários, uma vez que a estimulação e a preservação das funções cognitivas se encontram diretamente ligadas à qualidade de vida.

Palavras-chave: Envelhecimento. Deficiência intelectual. Reabilitação neuropsicológica.

\begin{abstract}
Conforming the increase of global population's life expectancy and due to several improvements in many society's sections, the ones with intellectual disability (ID) are also living more, the ones who until 30 years ago coulnd't live more than 15 years old. However, the fact that they are living more does not mean they are living better, because they are discriminated for their deficiency and also because they are elderly. In this especific population it is possible to observe the fact that they present early aging due to the commom illnesses in elderly in general population. With this overview there is also the lack of effective public policy which covers theses questions. The families and institutions, such as APAE, are aiming to make visible this aspects and demands, offering support and solutions to assit this public, with necessary care, including during the aging process. The neuropsychological rehabilitation is indicated as complement methodology to promote independency and authonomy of the recipients, once the estimulation and preservation of cognitive functions is directly connected with life quality.
\end{abstract}

Keywords: Aging. Intellectual deficiency. Neuropsychological rehabilitation. 


\section{INTRODUÇÃO}

Dados estatísticos das últimas décadas apontam a mudança no perfil etário da população mundial. Para o Brasil, esses estudos sugerem que em 2025 o país encontrar-se-á ocupando o $5^{\circ}$ lugar entre os países habitados por pessoas mais idosas, com aproximadamente 32 milhões com idade acima dos 60 anos (OLIVEIRA, 2013). Esse fato se explica por diversas razões: queda na mortalidade infantil; melhoria nas condições de saúde; ampliação de acessibilidade a benefícios; inclusão social, dentre outros fatores que influenciam diretamente a expectativa de vida da população, incluindo as pessoas com deficiência intelectual (DI) (CIPOLLA; LOPES, 2012).

Inúmeros indicadores internacionais demonstram o aumento expressivo na longevidade das pessoas com DI nos últimos 30 anos. Em 1947, a expectativa de vida de pessoas com Síndrome de Down era de 12-15 anos e, atualmente, não é raro encontrar idosos com a Síndrome, na idade de 6070 anos (BREITENBACH, 2011).

Porém, quando se fala em longevidade e ciclo de vida de pessoas com DI, o fato de estar vivendo mais não quer dizer exponencialmente que essas pessoas estejam com uma vida melhor (AGUIAR, 2018). A deficiência intelectual está historicamente ligada à segregação e à marginalização, acrescentando-se a ela o estereótipo da senilidade. Ou seja, a pessoa com DI está sujeita a vivenciar o duplo estigma: deficiente e idosa, mesmo com as garantias constitucionais e a ampla legislação em vigor (PORTELLA et al., 2015).

Na situação da Síndrome de Down, outro aspecto é considerado: o processo de envelhecimento das pessoas com esse diagnóstico pode iniciar-se precocemente, em comparação com o restante da população (ALVES, 2012). Por essa razão, é preciso considerar uma expectativa de vida diferenciada, com necessidades específicas de atenção à saúde (CASTRO, 2018), tendo em vista o surgimento de comorbidades que aceleram o declínio cognitivo e funcional da pessoa (FONSECA, et al., 2015).

Além disso, podem surgir problemas de saúde interligados ao processo de envelhecimento em idades inferiores, quando comparadas à população geral (GUILHOTO, 2014). O envelhecimento precoce é resultado de uma série de fatores, desde alterações metabólicas até a falta de programas e políticas públicas, baixa qualidade de serviços em assistência social e saúde, dentre outros aspectos (INSTITUTO APAE DE SÃO PAULO, 2013). 


\section{Apøe Ciênciø Artigo}

apaeciencia.org.br/revista

Desse modo, o envelhecimento de pessoas com DI requer especial atenção, não somente devido à questão dos efeitos da passagem natural do tempo, mas, principalmente, devido às demandas dessa fase da vida (FREITAS et al., 2010).

\section{PESSOAS COM DEFICIÊNCIA INTELECTUAL (DI) EM PROCESSO DE ENVELHECIMENTO}

A literatura demonstra inúmeros marcadores que podem sugerir o processo de envelhecimento precoce nas pessoas com DI a partir dos 30 ou 40 anos, em especial em pessoas com Síndrome de Down, com o aparecimento de determinadas doenças que geralmente são observadas em pessoas mais idosas, tais como: diminuição da aptidão física; obesidade; alterações comportamentais e psiquiátricas; epilepsia; doenças do coração não isquêmicas; osteoporose; doenças da pele; distúrbios da tireoide; alterações sensoriais (perdas visuais e auditivas); mudanças odontológicas ou doenças gastrointestinais, agravadas pelo uso da polifarmácia ao longo da vida (CARVALHO et al., 2015). No comprometimento cognitivo e funcional se evidenciam inúmeras dificuldades na execução das atividades de vida diária, com destaque especial para a atenção, as funções executivas e a memória, primordiais para o planejamento e o gerenciamento de ações e alcance de metas (AGUIAR, 2018).

Então, o binômio deficiência e envelhecimento tem impulsionado a busca de meios para uma proposta de cuidados que possa ser utilizada de maneira satisfatória para essa população, o que é considerado como um desafio para os envolvidos nesse processo, sejam profissionais ou familiares (FCEE, 2017).

$\mathrm{Na}$ deficiência intelectual se encontram limitações no funcionamento cognitivo, que estão associadas às limitações em diversas áreas adaptativas, como: atividades de vida diária, comunicação, adaptação social, funcionalidade acadêmica, de lazer e trabalho - em níveis diferenciados - durante toda a vida, demandando apoios que variam de parcial a total, nas diferentes tarefas executadas no cotidiano, interferindo diretamente na autonomia e independência (AGUIAR, 2018).

Os tipos relacionados ao cuidado são identificados conforme o nível de deficiência, visando a manutenção do bem-estar e a melhoria na qualidade de vida. Tais auxílios podem ser necessários 


\section{Apøe Ciênciø Artigo}

apaeciencia.org.br/revista

na realização de atividades que não conseguem ser realizadas pela pessoa sozinha ou estímulos específicos para que realizem tarefas para as quais tenham habilidades (ALMEIDA et al., 2016).

Muitas vezes, os cuidadores são membros da família, que assumem o papel de cuidador integral, voltando suas atividades para o bem-estar físico, a alimentação e a higiene, deixando para as instituições especializadas o papel de promover a integração social, a participação na comunidade e o desenvolvimento da autonomia (GIRARDI et al., 2012). Nas instituições, as atividades precisam ser ativas e dinâmicas, para oportunizar a criação de um ambiente rico, capaz de desenvolver as habilidades funcionais de pessoas com DI, buscando sua inclusão e participação na sociedade. Uma vez que o sujeito tem a oportunidade de explorar seu potencial, enriquecer o repertório para a solução de problemas cotidianos, adquire-se maior independência e autonomia (FCEE, 2017).

Outro ponto importante a ser considerado é a circunstância de adultos com DI morando com familiares que também necessitam de apoio e suporte, devido ao próprio processo de envelhecimento (AGUIAR, 2015). A convergência de envelhecimento entre os membros da família requer apoio e preparo para enfrentar o luto e a morte dentro das instituições, em conjunto com as famílias (FCEE, 2017). A Rede Apae se destaca como uma organização de renome e reconhecimento na área da inclusão social das pessoas com DI no Brasil. A primeira unidade apaeana foi criada em 1954, no Rio de Janeiro. Desde então, essa organização vem exercendo um papel relevante no atendimento desse público específico (MENDES, 2010).

A Rede Apae, pelas suas unidades localizadas em todas as regiões brasileiras, tem envidado esforços, junto ao poder legislativo, para alterar o Estatuto do Idoso, que estabelece a idade de 60 anos, e acima, como marco para o envelhecimento. Reivindica-se a inserção de um dispositivo legal que atenda às singularidades das pessoas com deficiência intelectual, visando à garantia dos seus direitos, no caso de precocidade. Desse modo, poderão usufruir de direitos e benefícios da população idosa de maneira antecipada, quando atestada pelos profissionais competentes (CIPOLLA; LOPES, 2012).

Em Santa Catarina, a FCEE (Fundação Catarinense de Educação Especial) adotou uma metodologia com uso de duas ferramentas, possibilitando o desenvolvimento de um trabalho efetivo junto à população que atende, de um modo geral. São eles: o Currículo Funcional Natural e a Classificação Internacional de Incapacidade, Funcionalidade e Saúde-CIF (OMS, 2004). Essa metodologia se estende às unidades apaeanas do estado. (FCEE, 2017) 


\section{Apøe Ciênciø Artigo}

apaeciencia.org.br/revista

O currículo funcional é uma proposta que aponta caminhos para o atendimento com auxílio da família e dos professores, que traçam objetivos do programa coletivamente, baseados nas necessidades atuais e futuras do sujeito. São identificados comportamentos e conhecimentos a adquirir, tendo em vista a sua relevância para promover independência e autonomia, adequando as atividades e as propostas de acordo com a idade cronológica e as singularidades da pessoa. Os programas são desenvolvidos de modo a tratar criança como criança, adolescente como adolescente e adulto como adulto (LE BLANC, 1992).

Quanto à CIF, trata-se de um sistema de classificação multiuso, desenvolvido para ser utilizado por diversos setores e disciplinas, com o objetivo de estudar e compreender a saúde e as suas relações com resultados de intervenções, alterações determinantes quanto à funcionalidade e saúde, por um modelo biopsicossocial da deficiência, que envolve o modelo social e o modelo médico para chegar a uma resposta que ofereça uma visão completa ao integrar as diferentes atuações da saúde: individual, biológica e social (CIF, 2004).

Porém, essas ferramentas, apesar dos bons resultados, necessitam de um método complementar, como a reabilitação neuropsicológica, que tem como objetivo uma atividade dinâmica para a readaptação dessa pessoa ao seu ambiente, por atividades com diversas estratégias e técnicas, considerando a plasticidade cerebral e as potencialidades do indivíduo. Em outras palavras, além de visar a melhoria cognitiva, objetiva-se otimizar e maximizar as aprendizagens em geral, considerando os problemas emocionais e comportamentais, para que a pessoa seja capaz de integrar-se ao meio, seja ele escolar, familiar e de trabalho, da maneira mais adequada possível (CORREA, 2009).

\section{REABILITAÇÃO NEUROPSICOLÓGICA PARA A PESSOA COM DI EM PROCESSO DE ENVELHECIMENTO}

A neuropsicologia é uma das atuações da psicologia em conjunto com a neurologia e o seu objeto de estudo se encontra na relação entre o cérebro e o comportamento humano. Tem como objetivo correlacionar as funções cognitivas com o funcionamento cerebral do indivíduo e, quando existe funcionamento deficiente de quaisquer das funções cognitivas (consequentemente, comprometimento de outras funções), atua na disfunção neuropsicológica existente no que tange à reabilitação neuropsicológica, como ocorre em pessoas com DI (CORREA, 2009) 


\section{Apøe Ciênciø Artigo}

apaeciencia.org.br/revista

A DI se caracteriza por limitações do funcionamento intelectual, em associação com, pelo menos, duas ou mais limitações em outras áreas de habilidades adaptativas também conhecidas como as AVDs (atividades de vida diária). As funções cognitivas desempenham papel fundamental nas atividades cotidianas, destacando-se a memória, a atenção e as funções executivas, que são de vital importância para a coordenação, o planejamento, bem como o gerenciamento das ações no alcance de metas (AGUIAR, 2018).

Para minimizar as defasagens no desempenho das funções cognitivas, propõem-se a oferta de intervenção por programas de reabilitação neuropsicológica, um caminho promissor não farmacológico, que objetiva amenizar as dificuldades nos setores funcionais, cognitivos e comportamentais das pessoas com DI, com base em técnicas cientificamente comprovadas e especificas, facilitando, dessa maneira, a sua inclusão na comunidade e a melhoria na sua qualidade de vida (CORRÊA, 2009).

Os objetivos da reabilitação neuropsicológica para as pessoas com DI se baseiam em capacitar pacientes e familiares para a convivência, reduzir e superar as limitações cognitivas, sociais e emocionais (SIMON; RIBEIRO, 2011), além de incentivar fatores de proteção cognitivos por estimulações do potencial individual para a promoção e a manutenção da autonomia e da independência (FONSECA et al., 2015). Desse modo, a finalidade da reabilitação é instigar os fatores protetivos da pessoa com DI, pela estimulação e pela busca do seu maior potencial. Entre outros fatores, é uma intervenção devida ao longo da vida e busca adaptação ao mercado de trabalho, socialização, prática de exercícios físicos e adoção de um estilo de vida ativo (FONSECA et al., 2015).

Estudos na área das neurociências, em especial na reabilitação neuropsicológica, demonstram estreitas relações entre qualidade de vida e funções cognitivas, o que faz dessa atuação interdisciplinar um campo promissor no desenvolvimento de ações para a promoção de um envelhecimento saudável das pessoas com DI (IRIGARAY et al., 2012).

Então, a oferta de programas como a reabilitação neuropsicológica para as pessoas com DI, em especial para as que se encontram em processo de envelhecimento pode vir a ser um caminho auspicioso na tentativa de minimizar os déficits no desempenho das funções cognitivas, uma vez que estudos já apontaram resultados positivos e melhoras significativas no desempenho, na manutenção e na prevenção do declínio cognitivo em outras populações (AGUIAR, 2018). 


\section{Apøe Ciênciø Artigo}

apaeciencia.org.br/revista

\section{CONCLUSÃO}

O tema deste ensaio é escasso em pesquisas, sob diferentes aspectos, no que tange ao envelhecimento, mais ainda quando se trata de pessoas com deficiência intelectual. Embora o tema seja de extrema importância e urgência, tem sido negligenciado. Da mesma maneira que o tema deficiência e envelhecimento não tem visibilidade, são ainda mais raros os estudos que fundamentam a oferta de atendimentos de qualidade para essa população específica.

Embora a reabilitação neuropsicológica seja uma das estratégias que podem vir a oferecer bons resultados, ainda tem um longo caminho a ser percorrido para que as muitas respostas existentes neste campo possam ser sanadas de maneira realmente efetivas. Pela escrita deste trabalho, fica registrada a emergência de novos estudos nessa área, a fim de promover ações realmente efetivas para as pessoas com DI, para que, além de viverem por mais tempo, possam ter uma vida digna, com qualidade, direitos e autonomia e para que possam realmente exercer seu papel de cidadãos na sociedade. 


\section{Apøe Ciênciø Artigo}

\section{REFERÊNCIAS}

AGUIAR, A. A. R. Avaliação neuropsicológica na deficiência intelectual: subsídios para um envelhecimento saudável. 2018. Disponível em: < http://www.editorarealize.com.br/revistas/cieh/trabalhos/TRABALHO_EV075_MD2_SA15_ID287 _25072017142203.pdf>. Acesso em: 10 jun. 2019.

AGUIAR, A. A. R. Deficiência Intelectual, envelhecimento e neurociência. Revista DI UFSCAR, São Paulo, v. 9, p. 30-35, jul/ago, 2015. Disponível em: < http://www.apaesp.org.br/ptbr/sobre-deficiencia-intelectual/publicacoes/PublishingImages/revista-di/artigos_pdf/DI_N9.pdf >. Acesso em: 13 jul. 2019.

ALMEIDA, M. A. A.; FALEIROS, V. P.; SANTOS, M. J. S.; HELDER, C. H. Representação social do cuidado e do cuidador familiar do idoso. Revista Katálysis, Florianópolis, v. 19, n. 1, p. 143-153, jan/jun, 2016. Disponível em: < http://www.scielo.br/scielo.php?pid=S141449802016000100143\&script=sci_abstract\&tlng=pt>. Acesso em: 12 jul. 2019.

ALVES, P. J. M. Estudo de follow-up do processo de envelhecimento de adultos com deficiência mental. 2012. 66 f. Dissertação (Mestrado Integrado em Psicologia) - Faculdade de Psicologia, Universidade de Lisboa, Lisboa, 2012. Disponível em: < http://repositorio.ul.pt/handle/10451/6906>. Acesso em: 12 jul. 2019.

BITTENCOURTE, Greicy Kelly; SILVA, Vilson Rodrigues da (Org.). Envelhecer com deficiência intelectual: aspectos básicos sobre o cuidado e o acesso aos direitos. Florianópolis: DIOESC; Secretaria de Estado da Educação - Fundação Catarinense de Educação Especial, 2017. Disponível em: < http://www.fcee.sc.gov.br/index.php/sala-de-imprensa/noticias/8858-cartilhasobre-deficiencia-intelectual-e-envelhecimento-ja-esta-disponivel-para-download $>$. Acesso em 12 jul. 019.

BREITENBACH, N. Paralelos em envelhecimento. 2011. Disponível em: <http://www.portaldoenvelhecimento.com/acervo/artieop/Geral/artigo2.htm>. Acesso em: 14 jul. 2019.

CARVALHO, C. L.; ARDORE, M.; CASTRO, L. R. Cuidadores familiares e o envelhecimento da pessoa com deficiência intelectual: implicações na prestação de cuidados. Revista Kairós Gerontologia, São Paulo, v. 18, n. 3, p. 333-352, jul/set 2015. Disponível em: < https://revistas.pucsp.br/index.php/kairos/article/view/27470>. Acesso em: 09 jul. 2019.

CIPOLA, M. A; LOPES, A. Envelhecimento e Associação de Pais e Amigos dos Excepcionais do município de São Paulo: uma caracterização dos serviços de atendimento a pessoa com deficiência intelectual. Kairós, São Paulo, v. 15, n. 6, p. 239-267, Revista Kairós Gerontologia 2012. Disponível em: < https://revistas.pucsp.br/index.php/kairos/article/viewFile/17306/12852>. Acesso em: 08 jul. 2019.

CORRÊA, R. C. R. Uma proposta de reabilitação neuropsicológica através do programa de enriquecimento instrumental (PEI). Ciências \& Cognição, Rio de Janeiro, v. 14, n. 2, p. 47-58, jul, 


\section{Apøe Ciênciø Artigo}

apaeciencia.org.br/revista

2009. Disponível em: < http://www.cienciasecognicao.org/revista/index.php/cec/article/view/7>. Acesso em: 2 jul. 2019.

FONSECA, L. M.; NAVATTA, A. C. R.; BOTTINO, C. M. C.; MIOTTO, E. C. Reabilitação cognitiva da demência em adultos com síndrome de Down. Revista DI, São Paul, n. 9, jul/ago 2015. Disponível em: < http://www.apaesp.org.br/pt-br/sobre-deficienciaintelectual/publicacoes/PublishingImages/revista-di/artigos_pdf/DI_N9.pdf >. Acesso em: 10 jul. 2019.

FREITAS, M. C. et. al. O significado da velhice e da experiência de envelhecer para os idosos. Revista da Escola de Enfermagem da USP, São Paulo, v. 44, n. 22, p. 407-12, 2010. Disponível em: < http://www.scielo.br/scielo.php?pid=S0080-

62342010000200024\&script=sci_abstract\&tlng=pt>.Acesso em: 07 jul. 2019.

GUILHOTO, L. M. de F. F.; LEONDARIDES, M. R. S. C.; CASTRO, L. R.; SENA, S., MAIZE, A.; ALVES, S.; ALMEIDA, C.; KARMELI, D.; MACEDO, E.; TARANDACH, E. R.; RIGHINI, J. B.; PREZIA, M. F. B.; FARIA, V.; CAVALHEIRO, E. A. Envelhecimento e Deficiência Intelectual na Grande São Paulo. DI - Deficiência Intelectual, São Paulo, v. 4. n. 7, jul/dez 2014. Disponível em: < https://apaebrasil.org.br/uploads/5386-artigo_envelhecimento.pdf> Acesso em: 08 jul. 2019.

GUILHOTO, Laura Maria de Figueredo Ferreira (Org.); MERLIN, Cynthia Helena (Coord.). Envelhecimento e deficiência intelectual: uma emergência silenciosa. 2. ed. São Paulo: Instituto APAEde São Paulo, 2013.

IRIGARAY, T. Q. et. al. Efeitos de um treinamento de atenção, memória e funções executivas na cognição de idosos saudáveis. Psicologia Reflexão e Critica, Porto Alegre, v. 25, n. 1, p. 188-202, 2012. Disponível em: < http://www.scielo.br/scielo.php?script=sci_arttext\&pid=S010279722012000100023>. Acesso em: 07 jul. 2019.

LEBLANC, J. M. Variables involucradas em la enseñanza grupal e individual para personas com retardo. In: SUPLINO, M. Currículo Funcional Natural: guia prático para educação na área de autismo e deficiência mental. 3. ed. Rio de Janeiro: Diferenças Editora, 2009.

MENDES, E. G. Breve histórico da educação especial no Brasil. Revista Educación y Pedagogia, Colômbia, v. 22, n. 57, p. 93-109, maio/ago, 2010. Disponível em: < https://aprendeenlinea.udea.edu.co/revistas/index.php/revistaeyp/article/viewFile/9842/9041>. Acesso em: 07 jul. 2019.

OLIVEIRA, A. F. Deficiência intelectual e envelhecimento: um desafio contemporâneo. APAECiência, Brasília, v. 1, n. 1, p. 33-43, jan/abr, 2013. Disponível em: < http://apaeciencia.org.br/index.php/revista/article/view/4>. Acesso em: 12 jul. 2019.

PORTELLA, M. R.; GIRARDI, M.; COLUSSI, E. L.; SANTOS, M. I. P. O.; SCORTEGAGNA, H. M. A pessoa deficiente intelectual e o envelhecimento: da percepção do fenômeno à realidade

Fed. Nac. das Apaes- Fenapaes $\mid$ Brasília/DF $\mid$ v.10| n² | P. 52 - $61 \quad$ jul./dez.2018 | ISSN 2237-4329 


\section{Apøe Ciênci@ Artigo}

apaeciencia.org.br/revista

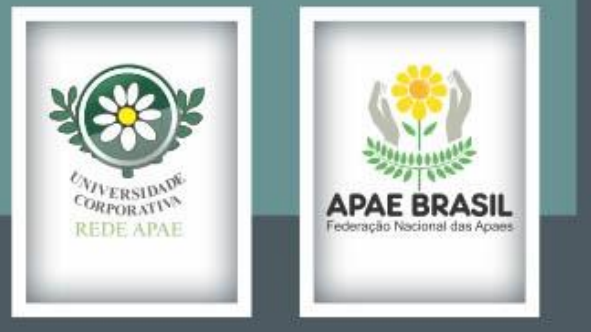

cotidiana. Revista Kairós Gerontologia, São Paulo, v. 18, n. 2, p. 401-420, 2015. Disponível em: < https://revistas.pucsp.br/index.php/kairos/article/viewFile/28180/19808>. Acesso em: 10 jul. 2019.

SIMON, S. S., RIBEIRO, M. P. O. Comprometimento cognitivo leve e a reabilitação neuropsicológica: uma revisão bibliográfica. Pisc.Rev. São Paulo, v. 20, n. 1, p. 93-122, 2011. Disponível em: < https://revistas.pucsp.br/index.php/psicorevista/article/viewFile/6795/4918>. Acesso em: 10 jul. 2019. 